\title{
Crecimiento postraumático, rumiación y estrategias de afrontamiento en nińos, nińas y adolescentes expuestos al terremoto de Coquimbo de 2015
}

\author{
Mariela Andrades ${ }^{1}$, Felipe E. García ${ }^{2}$ \\ Universidad Central ${ }^{1}$ - Chile, Universidad Santo Tomás ${ }^{2}$ - Chile
}

El objetivo del presente estudio fue identificar las estrategias de afrontamiento y estilos rumiativos que se relacionan con Crecimiento Postraumático (CPT), en una muestra de 105 participantes de 12 a 16 años, encuestados 12 meses después del terremoto del año 2015 en la ciudad de Coquimbo, Chile. Los resultados muestran que la rumiación deliberada y las estrategias de afrontamiento productivas predijeron significativamente el CPT. Además, se observó que cuando los participantes sienten amenazas importantes a su integridad, aumentan sus niveles de CPT. Estos resultados permitirían diseñar estrategias de intervención que ayuden a enfrentar las consecuencias de un desastre natural en niños, niñas y adolescentes.

Palabras clave: Desastre natural, pensamiento repetitivo, psicología positiva, severidad del evento, trauma

Posttraumatic growth, rumination and coping strategies in children and adolescents exposed to the 2015 Coquimbo earthquake

The aim of the present study was to identify the coping strategies and ruminative styles that are related to Posttraumatic Growth (PTG), in a sample of 105 participants from 12 to 16 years old, surveyed 12 months after the 2015 earthquake in the city of Coquimbo, Chile. The results show that deliberate rumination and productive coping strategies significantly predicted PTG. In addition, it was observed that when participants feel significant threats to their integrity, their PTG levels increase. These results would allow designing intervention strategies that help to cope the consequences of a natural disaster in children and adolescents.

Keywords: Natural disaster, repetitive thinking, positive psychology, severity of event, trauma

Crescimento pós-traumático, ruminação e estratégias de enfrentamento em crianças e adolescentes expostos ao terremoto de Coquimbo 2015

O objetivo do presente estudo foi identificar as estratégias de enfrentamento e os estilos ruminativos relacionados ao Crescimento Pós-Traumático (CPT), em uma amostra de 105 participantes de 12 a 16 anos, pesquisados 12 meses após o terremoto de 2015 na cidade

1 Doctora en Psicología. Escuela de Psicología, Universidad Central, Chile. Gonzalo Hernández Uribe Building, 414 San Ignacio, Santiago, Chile. CP: 8330508. Email: marielaandrades@ hotmail.com

2 Doctor en Psicología. Facultad de Ciencias Sociales y Comunicaciones, Universidad Santo Tomás, Chile. Av. Prat 855, Concepción, Chile. CP: 4030000. Email: felipegarciam@yahoo.es 
de Coquimbo. Chile. Os resultados mostram que a ruminação deliberada e estratégias de enfrentamento produtivas previram significativamente o TCP. Além disso, observou-se que quando os participantes sentem ameaças significativas à sua integridade, seus níveis de CPT aumentam. Estes resultados permitiriam desenhar estratégias de intervenção que ajudem a enfrentar as consequências de um desastre natural em crianças e adolescentes.

Palavras-chave: desastre natural, pensamento repetitivo, psicologia positiva, gravidade do evento, trauma

Croissance post-traumatique, rumination et «stratégies d'adaptation» chez les enfants et les adolescents exposés au séisme de Coquimbo de 2015

L'objectif de la présente étude était d'identifier les stratégies d'adaptation et les styles ruminatifs liés à la croissance posttraumatique (CPT), sur un échantillon de 105 participants âgés de 12 à 16 ans, enquêtés 12 mois après le séisme de 2015 à Coquimbo. , Chili. Les résultats montrent qu'une rumination délibérée et des stratégies d'adaptation productives ont permis de prédire de manière significative le CPT. En outre, il a été observé que lorsque les participants ressentaient des menaces importantes pour leur intégrité, leurs niveaux de CPT augmentaient. Ces résultats permettraient de concevoir des stratégies d'intervention permettant de faire face aux conséquences d'une catastrophe naturelle chez les enfants et les adolescents.

Mots clés: catastrophe naturelle, pensée répétitive, psychologie positive, gravité de l'événement, traumatisme 


\section{Introducción}

$\mathrm{Al}$ atardecer del 16 de septiembre de 2015 tuvo lugar en Coquimbo, Chile, un terremoto grado 8,4 en la escala de magnitud local Richter. Esta catástrofe costó la vida a 13 personas, dejó a casi 30.000 personas damnificados y 1.100 viviendas quedaron destruidas o con daño severo (United States Geological Survey, 2015).

Desde el punto de vista del impacto psicológico, los terremotos son eventos sorpresivos, intensos e incontrolables, y ponen en riesgo la integridad física y psicológica de las personas que los experimentan (Leiva, 2011). Un evento como este pone a prueba la capacidad de adaptación pudiendo alterar la salud mental de las personas tanto en el corto como a largo plazo (Rochanakorn, 2007), incluyendo tanto a personas adultas como a niños, niñas y adolescentes (Kun et al., 2009).

Las respuestas emocionales de los niños, niñas y adolescentes a estos desastres pueden variar desde alteraciones mínimas y de corta duración (Cohen \& Gadassi, 2009), hasta la aparición de respuestas psicopatológicas, entre ellas, el estrés postraumático (Alisic \& Kleber, 2010; Norris \& Elrod, 2006) ya sea en forma de trastorno (TEPT) o sintomatología (SPT). Sin embargo, se ha demostrado que también los niños, niñas y adolescentes podrían resistir con entereza situaciones desfavorables (Alisic et al., 2008), incluso impulsando un aprendizaje o una percepción de cambios positivos luego de una crisis vital importante, respuesta denominada Crecimiento Postraumático (CPT; Calhoun \& Tedeschi, 1999).

El CPT es conceptualizado como la experiencia de cambios positivos que una persona experimenta como resultado del proceso de lucha que emprende a partir de la vivencia de un suceso traumático (Calhoun \& Tedeschi, 1999). Puede manifestarse a través de cambios en uno mismo, fortalecimiento en las relaciones interpersonales y cambios en la filosofía de vida y la espiritualidad (Calhoun et al., 2010). 
Si bien el CPT se ha estudiado principalmente en adultos (i.e., Siqveland et al., 2015), se ha sugerido que el CPT es posible entre poblaciones más jóvenes (Meyerson et al., 2011). Existe evidencia de algún nivel de CPT a partir de los 6 ańos de edad, después de un evento potencialmente traumático (Cryder et al., 2006).

Entre estos eventos potencialmente traumáticos para la población infantil, se encuentran las enfermedades que amenazan la vida (Barakat et al., 2006), accidentes de tránsito (Salter $\&$ Stallard, 2004), violencia en la comunidad (Park \& Ai, 2006), desastres naturales (Cryder et al., 2006; Hafstad et al., 2010; Kilmer \& Gil-Rivas 2010; Yu et al., 2010) y otros acontecimientos (Alisic et al., 2008; Taku et al., 2009).

Los factores que determinan que una experiencia traumática pueda generar CPT son diversos (Cho \& Park, 2013). Siendo uno de los aspectos a considerar, la manera en que la persona afronta la situación adversa, debido a que se relaciona con la forma de manejar el estrés (Zoellner \& Maerker, 2006).

Lazarus (1966), define el afrontamiento como un proceso que se activa cuando se percibe una amenaza, y que tiene como objetivo regular el conflicto emocional y eliminar dicha amenaza. Lazarus y Folkman (1984) señalan que este afrontamiento surge en la interacción de la persona con su entorno. A su vez, las estrategias de afrontamiento son el conjunto de respuestas (sentimientos, acciones, pensamientos) que un individuo utiliza para resolver situaciones problemáticas y reducir las tensiones generadas por ellas (Casullo \& Fernández, 2001). Las estrategias pueden ser productivas, centradas en modificar la fuente del problema, mediante la búsqueda de solución activa, o improductivas, dirigidas a reducir la emoción (Morales et al., 2012).

Entre las estrategias de afrontamiento productivas se encuentran la solución activa, búsqueda de información, comunicar el problema y la actitud positiva (Morales et al., 2012). Estas estrategias, se asociaron a un desarrollo emocional saludable para aquellos nińos y nińas que la emplean (Compas et al., 2001). Por otra parte, entre las estrategias improductivas se encontrarían la evitación cognitiva, evitación conductual, indiferencia, reservarse el problema y conducta agresiva (Morales 
et al., 2012). Estas estrategias se asocian en la infancia con menor bienestar psicológico y problemas de adaptación (González et al., 2002).

La relación entre CPT y estrategias de afrontamiento en niños, niñas y adolescente ha sido escasamente estudiada. Se ha encontrado, por ejemplo, que el uso de estrategias de afrontamiento productivas y de aceptación en niños y niñas con cáncer predijeron niveles más altos de CPT que aquellos que presentaron estrategias de afrontamiento evitativas (Turner-Sack et al., 2012).

Otro factor relacionado con el CPT es la rumiación, la que alude a diferentes formas de pensamientos repetitivos. Calhoun y Tedeschi (2006) han sugerido la existencia de dos tipos de respuestas rumiativas después de un evento traumático: la rumiación intrusiva y la rumiación deliberada. La rumiación intrusiva implica que pensamientos no deseados sobre el evento irrumpen en el mundo cognitivo de la persona. La rumiación deliberada supone un esfuerzo más premeditado y consciente centrado en el manejo de la situación, que también puede permitir el volver a examinar el evento de forma voluntaria para tratar de entender lo sucedido.

En estudios con población adulta, se ha observado que tanto la rumiación intrusiva como deliberada se activan cuando una persona percibe un evento como estresante, lo que puede conducir a CPT (Alzugaray et al., 2015; García et al., 2017). Mientras la rumiación intrusiva parece estar especialmente asociada a sintomatología postraumática (Alzugaray et al., 2015); la presencia de rumiación más deliberada tiende a estar relacionada con CPT (Calhoun et al., 2010; Tedeschi \& Calhoun, 2004; García et al., 2017).

En el caso de niños, niñas y adolescentes, se ha observado que, al igual que en adultos, tanto la rumiación intrusiva como deliberada son centrales en el desarrollo del CPT (Kilmer et al., 2009; Andrades et al., 2018). Por otra parte, la rumiación deliberada fue el único predictor significativo del CPT, un año después del huracán Katrina (Kilmer \& Gil-Rivas, 2010).

La severidad del evento parece también influir en el CPT. El metaanálisis de Helgeson et al. (2006) encontró que una mayor gravedad 
objetiva $(r=0,07)$ y mayor estrés percibido $(r=0,14)$ se asociaban positivamente a un mayor CPT. La relación entre severidad y CPT sería explicable por cuanto es la gravedad percibida del suceso la que activa mecanismos que permiten el crecimiento (Calhoun $\&$ Tedeschi, 1999). Estos mecanismos serían los procesos de rumiación sobre el evento (Calhoun \& Tedeschi, 2006). Si bien hay pocas investigaciones sobre rumiación y CPT en niños, niñas y adolescentes, los estudios disponibles muestran que, al igual que en adultos, tanto la rumiación intrusiva como deliberada son centrales en el desarrollo del CPT, no obstante, la que presenta una relación más fuerte es la rumiación deliberada (Andrades et al., 2018; Kilmer \& Gil-Rivas, 2010).

Considerando la frecuencia de desastres naturales en Chile y la escasa investigación sobre sus efectos en la salud mental de niños, niñas y adolescentes, surge el interés de realizar la presente investigación, cuyo objetivo es identificar las estrategias de afrontamiento y estilos rumiativos que se relacionan con CPT, en niños, niñas y adolescentes que estuvieron expuestos al terremoto de Coquimbo, Chile, el año 2015.

\section{Método}

\section{Diseño de investigación}

El presente estudio utiliza un diseño de investigación cuantitativo, descriptivo y correlacional. Los datos fueron tomados en un solo corte temporal, por lo que corresponde a un estudio transversal.

\section{Participantes}

Se evaluó a 105 niños, niñas y adolescentes (51\% hombres y 49\% mujeres), en edades comprendidas entre los 12 y los 16 años $(M=$ 14.35; $D E=1.32$ ), que estuvieron expuestos al terremoto ocurrido en la ciudad de Coquimbo, en la zona norte de Chile, en septiembre de 2015. 


\section{Instrumentos}

Crecimiento postraumático. Fue medido con la escala PTGI-C-R (Kilmer et al. 2009; traducción de Andrades et al., 2016). Consta de 10 ítems que se responden a través de una escala Likert desde 0 (ningún cambio) a 3 puntos (mucho). En su versión chilena (Andrades et al., 2016) se evaluó su validez de constructo, encontrándose dos factores correlacionados y una consistencia interna de .84 para la escala total. En el presente estudio, se obtuvo una consistencia interna de $\alpha=.94$.

Rumiación: Fue medida a través de la Rumination Scale for Children (Cryder et al. 2006; traducción de Andrades et al., 2018), una escala de cinco ítems que evalúa la rumiación intrusiva y deliberada. Se utiliza una escala tipo Likert de 0 ("no pienso en esto") a 3 puntos ("pienso mucho en esto"). Ha sido aplicada en población infantil afectada por desastres naturales; su traducción mostró contar con validez de contenido (Andrades et al., 2018). En el presente estudio se obtuvo un $\alpha=.94$ para rumiación deliberada y .71 para rumiación intrusiva.

Severidad de la exposición a un evento traumático. Fue medido a través Exposure to Traumatic Events Questionnaire (Vernberg et al., 1996; traducido y adaptado para su uso en terremotos por Andrades et al., 2018). Consiste en 17 ítems dicotómicos (sí/no), de los cuales un ítem se refiere a la percepción directa de amenaza a la propia vida del niño, seis ítems están relacionados a eventos amenazantes específicos que pudieron observarse en el terremoto (por ejemplo, ruptura de puertas y ventanas) y diez ítems relacionados a experiencias disruptivas y pérdidas posdesastre (por ejemplo, cambiarse de hogar). El instrumento recoge experiencias específicas relacionadas a la exposición y su suma simple es un indicador de la severidad de la exposición. Ha sido utilizado en un estudio con niños expuestos a terremoto y mostró una correlación fuerte con síntomas de estrés postraumático $(r=.49)$. En el presente estudio, se obtuvo un $\alpha=.80$.

Estrategias de afrontamiento. Se utilizó la Escala de Afrontamiento para Niños (Morales et al., 2012). Esta escala evalúa estrategias de afrontamiento improductivas: indiferencia, conducta agresiva, reservarse el problema para sí, evitación cognitiva y evitación conductual; y 
estrategias de afrontamiento productivas: solución activa, comunicar el problema a otros, búsqueda de información y guía y actitud positiva. Son 35 ítems que se responden en una escala Likert de 0 (nunca) a 2 puntos (muchas veces). Sus autores evaluaron su validez de constructo encontrando dos factores de segundo orden, coincidentes con las estrategias productivas e improductivas; además, se evaluó su validez de criterio encontrándose asociación con variables relevantes para la salud y ajuste psicológico en la infancia (Morales et al., 2012). En el presente estudio se obtuvieron consistencias internas adecuadas para las estrategias productivas $(\alpha=.87)$ e improductivas $(\alpha=.78)$.

Cuestionario sociodemográfico. Se construyó un cuestionario que incluyó preguntas como la edad, sexo, ciudad de residencia en el momento del terremoto y ciudad de residencia actual.

\section{Procedimiento}

La recolección de datos fue realizada doce meses después del terremoto. Para ello, se estableció contacto con organizaciones sociales de la zona afectada, quienes proporcionaron datos de establecimientos educacionales. La elección de los cursos fue al azar. Se aplicaron los cuestionarios a los niños, niñas y adolescentes en sus respectivas salas de clases, realizando una aplicación grupal asistida por estudiantes de pre-grado, acompańados por la respectiva profesora de cada curso.

Previamente, se informó a los padres y se solicitó su consentimiento. La participación de los niños, niñas y adolescentes fue de carácter voluntario, sus identidades se manejaron con discreción y se garantizó la confidencialidad de los datos entregados, los que solo fueron analizados en su conjunto. Este estudio fue aprobado en sus aspectos éticas por una comisión de la Escuela de Psicología de la Universidad Central.

\section{Análisis de datos}

En primer lugar se realizaron análisis descriptivos de las variables de estudio. Luego se evaluó la relación entre las variables utilizando la 
correlación $r$ de Pearson y la Regresión Lineal Múltiple. Así mismo, se evaluó si existían diferencias en sexo en las variables de estudio usando $t$ de student.

El tratamiento estadístico de los datos se realizó a través del programa Statistical Package for the Social Sciences (SPSS) versión 22.0.

\section{Resultados}

En primer lugar, se establecieron los estadísticos descriptivos y confiabilidad de los instrumentos usados en el estudio. Se puede observar en la tabla 1 que la curtosis y asimetría se encuentran en todas las variables en el rango \pm 2 , valor sugerido para considerar la distribución de los datos como paramétrica (Bollen \& Long, 1994).

\section{Tabla 1}

Estadisticos descriptivos de los instrumentos $(n=105)$

\begin{tabular}{lcccccc}
\hline & Mín & Máx & Media & DE & Asimetría & Curtosis \\
\hline Rumiación intrusiva & 0 & 4 & 1.15 & 1.30 & .94 & -.26 \\
Rumiación deliberada & 0 & 9 & 4.42 & 3.38 & .08 & -1.45 \\
EA-Indiferencia & 0 & 8 & 2.22 & 2.05 & .90 & .37 \\
EA-Conducta Agresiva & 0 & 8 & 2.67 & 1.89 & .29 & -.37 \\
EA-Reservarse el & 0 & 8 & 4.03 & 2.11 & .16 & -.32 \\
$\begin{array}{l}\text { Problema } \\
\text { EA-Evitación Cognitiva }\end{array}$ & 0 & 6 & 2.80 & 1.35 & -.20 & -.09 \\
EA-Evitación & 0 & 7 & 3.54 & 1.73 & -.12 & -.06 \\
$\begin{array}{l}\text { Conductual } \\
\text { EA-Solución Activa }\end{array}$ & 0 & 8 & 4.30 & 1.92 & -.11 & -.66 \\
$\begin{array}{l}\text { EA-Comunicar el } \\
\text { Problema }\end{array}$ & 0 & 8 & 3.15 & 2.44 & .57 & -.54 \\
$\begin{array}{l}\text { EA-Búsqueda de } \\
\text { Información }\end{array}$ & 0 & 8 & 3.43 & 2.50 & .37 & -.86
\end{tabular}




\begin{tabular}{lcccccc}
\hline & Mín & Máx & Media & DE & Asimetría & Curtosis \\
\hline EA-Actitud Positiva & 0 & 8 & 4.73 & 1.94 & .06 & -.42 \\
EA-Improductivo $\dagger$ & 1 & 32 & 15.26 & 5.50 & .25 & .78 \\
$\begin{array}{l}\text { EA-Productivo } \dagger \\
\begin{array}{l}\text { Crecimiento } \\
\text { postraumático }\end{array}\end{array}$ & 0 & 31 & 15.61 & 6.72 & .02 & -.70 \\
\hline
\end{tabular}

$\mathrm{EA}=$ estrategia de afrontamiento; $\dagger=$ estrategia de segundo orden

Se revisaron también las frecuencias de SI/NO en las respuestas al cuestionario de exposición al trauma, con la finalidad de observar cómo habían vivenciado el sismo los participantes. Podemos observar, por ejemplo, que casi la mitad de los niños, niñas y adolescentes pensó durante el terremoto que podía morir (ver tabla 2)

\section{Tabla 2}

Frecuencias y porcentaje de niños, niñas y adolescentes en relación a cada pregunta del Cuestionario de Exposición a Eventos Traumáticos $(n=105)$.

En las últimas 2 semanas...

$\begin{array}{lc}\mathrm{NO} & \mathrm{SI} \\ n(\%) & n(\%)\end{array}$

1. ¿En algún momento durante el terremoto, $58(55.2 \%) \quad 47(44.8 \%)$ pensaste que podrías morir?

2. ¿Se rompieron las ventanas o puertas en el $89(84.8 \%) \quad 16(15.2 \%)$ lugar que estuviste durante el terremoto?

3. ¿Te lesionaste durante el terremoto? $102(97.1 \%) \quad 3(2.9 \%)$

4. ¿Viste a alguien herido gravemente durante el terremoto?

$99(94.3 \%) \quad 6(5.7 \%)$

5. ¿Tu mascota o alguna mascota que te gustaba $98(93.3 \%) \quad 7(6.7 \%)$ fue herida o murió durante el terremoto?

6. ¿¡Fuiste golpeado por algo durante el $89(84.8 \%) \quad 16(15.2 \%)$ terremoto? 
En las últimas 2 semanas... $\quad$ NO $\quad$ SI

$n(\%) \quad n(\%)$

7. ¿Tuviste que salir fuera de la casa durante el $78(74.3 \%) \quad 27(25.7 \%)$ terremoto debido a que la vivienda en la que estabas fue muy dañada?

8. ¿Fue tu casa dańada o destruida por el $\quad 86(81.9 \%) \quad 19(18.1 \%)$ terremoto?

9. ¿Tu ropa o juguetes fueron destruidos por el $93(88.6 \%) \quad 12(11.4 \%)$ terremoto?

10. ¿ Ha sido difícil ver a tus amigos después $\quad 95$ (90.5\%) 10 (9.5\%) del terremoto, porque se trasladaron o tú debiste cambiarte de casa?

11. ¿Tú o tu familia tuvieron problemas para $94(89.5 \%) \quad 11(10.5 \%)$ conseguir suficiente comida o agua después del terremoto?

12. ¿Te mudaste a un nuevo lugar debido al $\quad 101(96.2 \%) \quad 4(3.8 \%)$ terremoto?

13. ¿Tuviste que ir a una nueva escuela debido $100(95.2 \%) \quad 5(4.8 \%)$ al terremoto?

14. ¿Tuviste que vivir lejos de tus padres durante $102(97.1 \%) \quad 3(2.9 \%)$ una semana o más debido al terremoto?

15. ¿Alguien ha robado algo de tu casa desde $102(97.1 \%) \quad 3(2.9 \%)$ el terremoto?

16. ¿ंAlguno de tus padres perdió su trabajo $\quad 101(96.2 \%) \quad 4(3.8 \%)$ debido al terremoto?

17. ¿Tu mascota escapó o tuvo que ser regalada $90(85.7 \%) \quad 15(14.3 \%)$ debido al terremoto?

Al evaluar la correlación bivariada de las variables dependientes con el CPT a través del estadístico de correlación $r$ de Pearson (ver tabla $3)$. Se observa que la relación más fuerte la tiene con rumiación deliberada $(r=.90)$, seguido de las estrategias de búsqueda de información $(r=.69)$, comunicar el problema $(r=.64)$ y estrategias productivas $(r=$ $.64)$, entre otras, esta última de segundo orden. 


\section{Tabla 3}

Correlaciones bivariadas de orden cero entre las variables del estudio y el crecimiento postraumático $(n=105)$

\begin{tabular}{lc}
\hline & $\begin{array}{c}\text { Crecimiento } \\
\text { postraumático }\end{array}$ \\
\hline Rumiación intrusiva & $.42^{* * *}$ \\
Rumiación deliberada & $.90^{* * *}$ \\
EA-Indiferencia & $-.43^{* * *}$ \\
EA-Conducta Agresiva & -.06 \\
EA-Reservarse el Problema & -.07 \\
EA-Evitación Cognitiva & .11 \\
EA-Evitación Conductual & $.34^{* * *}$ \\
EA-Solución Activa & $.21^{*}$ \\
EA-Comunicar el Problema & $.64^{* * *}$ \\
EA-Búsqueda de Información & $.69^{* * *}$ \\
EA-Actitud Positiva & $.33^{* * *}$ \\
EA -Improductivo $\dagger$ & -.07 \\
EA-Productivo $\dagger$ & $.64^{* * *}$ \\
\hline
\end{tabular}

$\mathrm{EA}=$ estrategia de afrontamiento; $\dagger$ estrategia de segundo orden

Se evaluó la relación entre las preguntas del cuestionario de severidad del evento con el crecimiento postraumático utilizando para ello la $t$ de student para muestras independientes. Los resultados se pueden observar en la tabla 4, en el que se han omitido los reactivos en los que no se encontraron diferencias significativas. Se pude visualizar en ella que cuando los nińos, niñas y adolescentes sienten amenazas importantes a su integridad, aumentan sus niveles de CPT que cuando estas amenazas no existen. Entre esas amenazas se encuentran pensar que se puede morir, ver a alguien herido, ver a una mascota afectada, ser golpeado por algo, etc. 


\section{Tabla 4}

Comparación entre las respuestas (Si/No) a los distintos reactivos de severidad y el crecimiento postraumático, media, desviación estándar, valor ty valor $p(n=105)$

En las últimas 2 semanas...

$$
\text { SÍ }
$$

$\mathrm{NO}$

$$
M(D E)
$$

1. ¿En algún momento durante el

$19.40(8.40)$

$11.55(8.64)$

$4.689<.001$ terremoto, pensaste que podrías morir?

4. ¿Viste a alguien herido gravemente durante el terremoto?

5. ¿Tu mascota o alguna mascota que te gustaba fue herida o murió durante el terremoto?

6. ¿Fuiste golpeado por algo durante el terremoto?

10. ¿Ha sido difícil ver a tus amigos después del terremoto, porque se trasladaron o tú debiste cambiarte de casa?

11. ¿Tú o tu familia tuvieron problemas para conseguir suficiente comida o agua después del terremoto?

14. ¿Tuviste que vivir lejos de tus padres durante una semana o más debido al terremoto?

15. ¿Alguien ha robado algo de tu casa desde el terremoto?

16. ¿Alguno de tus padres perdió su trabajo debido al terremoto?

$23.00(7.21) \quad 14.59(9.28) \quad 2.178 \quad .032$

$22.43(10.06) \quad 14.43(9.12) \quad 2.227 \quad .028$

$19.31(8.98) \quad 14.30(9.26) \quad 2.000 \quad .048$

$24.30(6.50) \quad 14.09(9.10) \quad 4.521 \quad .001$

$23.27(5.87) \quad 14.01(9.23) \quad 4.563 \quad<.001$

$27.00(3.00) \quad 14.72(9.25) \quad 6.270 \quad .006$ $25.67(6.66) \quad 14.75(9.26) \quad 2.021 \quad .046$ $27.75(2.63) \quad 14.56(9.17) \quad 8.238 \quad<.001$ 
A continuación se realizó una Regresión Lineal Múltiple, considerando como predictores de CPT a la rumiación intrusiva, rumiación deliberada, estrategias productivas, estrategias improductivas y severidad del evento. El modelo predictivo resultó significativo $\left(F_{(5,97)}=\right.$ $88.990, p<.001)$, con un $\mathrm{R}^{2}=.82$. El modelo de regresión se observa en la tabla 5 y en ella se observa que las variables que predicen CPT son la rumiación deliberada $(\beta=.75)$ y las estrategias de afrontamiento productivas $(\beta=.15)$.

\section{Tabla 5}

Regresión Lineal Múltiple para la predicción de crecimiento postraumática $(n=105)$

\begin{tabular}{lccccc}
\hline \multirow{2}{*}{$\begin{array}{l}\text { Variable predictora } \\
\end{array}$} & \multicolumn{7}{c}{$\begin{array}{c}\text { Coeficientes no } \\
\text { estandarizados }\end{array}$} & $\begin{array}{c}\text { Coeficientes } \\
\text { tipificados }\end{array}$ & $\begin{array}{c}\text { Valor } \\
\text { B }\end{array}$ & $\begin{array}{c}\text { Valor } \\
\text { ET }\end{array}$ & $\beta$ & & $p$ \\
\hline (Constante) & 2.58 & 1.43 & & 1.808 & .074 \\
Rumiación intrusiva & .55 & .36 & .07 & 1.524 & .131 \\
Rumiación deliberada & 2.08 & .18 & .75 & 11.572 & .000 \\
$\begin{array}{l}\text { Estrategias de afrontamiento } \\
\text { improductivas }\end{array}$ & -.06 & .08 & -.04 & -.824 & .412 \\
$\begin{array}{l}\text { Estrategias de afrontamiento } \\
\text { productivas }\end{array}$ & .20 & .08 & .15 & 2.483 & .015 \\
Severidad del evento & .23 & .18 & .06 & 1.325 & .188 \\
\hline
\end{tabular}

Finalmente se hace una comparación por sexo (mujer/hombre) en relación al CPT. Se encontraron diferencias significativas $(t=4.321$; $p<.001)$, obteniendo las mujeres $(M=18.96 ; D E=8.15)$ mayores puntajes de CPT que los hombres $(M=11.63 ; D E=9.08)$. 


\section{Discusión}

El objetivo de la presente investigación fue identificar las estrategias de afrontamiento y estilos rumiativos que se relacionan con CPT, en niños, niñas y adolescentes que estuvieron expuestos al terremoto ocurrido en la ciudad de Coquimbo, Chile, el año 2015.

Uno de los resultados interesantes observados en el estudio dice relación a la percepción de amenaza manifestada por los participantes durante el terremoto, lo que se refleja en el alto porcentaje (45\%) que contestó positivamente al ítem "en algún momento durante el terremoto, pensaste que podrías morir" y que supera holgadamente a los otros ítems de exposición. Esto da pie a considerar al terremoto como un evento potencialmente traumático para ellos y con un posible efecto en su salud mental (La Greca \& Prinstein, 2002), lo que habría activado los procesos psicológicos que fueron objeto de evaluación en el presente estudio, como las estrategias de afrontamiento, los procesos rumiativos y el crecimiento postraumático

El análisis de correlaciones indicó que la rumiación deliberada, seguido de las estrategias de búsqueda de información, comunicar el problema y el uso general de estrategias productivas mostraron una relación más fuerte con CPT. Estos resultados se vieron corroborados en la regresión lineal múltiple, en la cual la rumiación deliberada y las estrategias productivas mostraron ser predictores significativos de CPT.

La relación entre rumiación deliberada y CPT es concordante con otros estudios en niños, niñas y adolescentes (Andrades et al., 2018; Kilmer et al., 2009; Kilmer \& Gil-Rivas, 2010) y podría deberse a que la rumiación deliberada conlleva una búsqueda voluntaria para dar sentido a lo ocurrido, pudiendo ser relevante, por tanto, en el cambio de las creencias y esquemas cognitivos de quienes sufren eventos adversos (Cann et al., 2011).

Por otro lado, si bien los estudios que abordan la relación entre estrategias de afrontamiento y CPT en niños, niñas y adolescentes es escasa (Turner-Sack et al., 2012), se observan en el presente estudio resultados similares a los encontrados en población adulta (Linley \& 
Joseph, 2004). Las estrategias de búsqueda de información, comunicar el problema y el uso general de estrategias productivas se relacionan a un mayor CPT que aquellos que presentan estrategias de afrontamiento improductivas. Las estrategias productivas son similares a las estrategias centradas en el problema de los estudios con personas adultas, e implican que los niños, niñas y adolescentes mantienen un sentido de control que los lleva a enfrentar las consecuencias del evento estresor y no evadirlos, lo que facilitaría la auto-percepción de cambios positivos, una de las dimensiones del CPT. La evidencia muestra que la búsqueda de información y comunicar el problema son consideradas por algunos autores, como estrategias funcionales que promueven la salud mental (Dávila $\&$ Guarino, 2001). Las estrategias de afrontamiento de aproximación al problema se asocian a resultados favorables de adaptación socioemocional en la infancia (Hampel \& Petermann, 2006; Wright et al., 2010).

Junto a esto, los resultados dan cuenta que cuando los participantes sienten amenazas importantes a su integridad, aumentan sus niveles de CPT. Esta relación sería explicable por cuanto es justamente la gravedad percibida del suceso la que activa mecanismos que permiten el CPT (Calhoun \& Tedeschi, 1999). Estos mecanismos, según algunos autores (Calhoun \& Tedeschi, 2006), serían los procesos de rumiación sobre el evento, pues probablemente la propia gravedad del evento lleve a un fuerte cuestionamiento de creencias, en forma de pensamiento repetitivo, lo que propiciaría el crecimiento (Páez et al., 2011).

Respecto al sexo, se observan diferencias significativas, presentando las mujeres una mayor puntuación de CPT que los hombres. Según algunos autores (Janoff-Bulman, 2006; Tedeschi \& Calhoun, 2004; Vishnevsky et al., 2010), esto podría deberse a diferencias en las estrategias de afrontamiento y en el tipo de rumiación utilizadas, con una mayor conciencia de las fortalezas personales y apreciación de la importancia de las conexiones sociales por parte de las mujeres. En población infantil, estas diferencias aún se encuentran en estudio, sin embargo, algunos investigaciones han arrojado mayor puntuación de CPT en mujeres, aunque estas diferencias no han sido significativas (Cryder et al., 2006) 
Este estudio presenta algunas limitaciones a considerar. Una de ellas es su naturaleza transversal, que impide establecer relaciones causaefecto entre las variables independientes y el CPT observado en los niños, niñas y adolescentes. En segundo lugar, la selección de los participantes fue intencional y su tamaño fue pequeño, por lo que la generalización de estos resultados debe tomarse con cuidado. Por último, si bien se incorporaron variables importantes según la literatura para explicar el CPT en niños, niñas y adolescentes, habría sido relevante la incorporación de otras variables que contribuirían a una mejor comprensión de los mecanismos que actúan sobre la salud mental de los niños, niñas y adolescentes, como el apoyo social (Schroevers et al., 2010) o el estado emocional de sus padres (Kilmer et al., 2009).

El presente estudio aporta a establecer predictores de CPT, ya que descubrir qué procesos psicológicos conducen al CPT en niños, niñas $\mathrm{y}$ adolescentes afectados por desastres naturales resulta relevante para perfilar estrategias de intervención clínica, orientada a la búsqueda de significados asociados al evento y crecer con la experiencia traumática (González, 2004). Por ejemplo, se plantea el desafío de promover un tipo de procesamiento cognitivo y estrategias de afrontamiento que promueven el crecimiento, a través de estrategias de intervención orientadas a estimular un pensamiento más voluntario y constructivo y una forma de actuar más productivo para enfrentar las consecuencias de un desastre natural en los niños, niñas y adolescentes.

\section{Referencias}

Alisic, E. \& Kleber, R. J. (2010). Measuring posttraumatic stress reactions in children: Preliminary validation of the Children's Responses to Trauma Inventory. Journal of Child and Adolescent Trauma, 3, 192-204. https://doi.org/10.1080/19361521.2010. 500979

Alisic, E., Van der Schoot, T., Van Ginkel, J. \& Kleber, R. (2008). Looking beyond posttraumatic stress disorder in children: 
Posttraumatic stress reactions, posttraumatic growth, and quality of life in a general population sample. The Journal of Clinical Psychiatry, 69(9), 1455-61. https://doi.org/10.4088/ JCP.v69n0913

Alzugaray, C., García, F., Reyes, A. \& Álvarez, R. (2015). Propiedades psicométricas de una versión breve de la escala de rumiación relacionada a un evento en población chilena afectada por eventos altamente estresantes. Ajayu, 13(2), 183-198.

Andrades, M., García, F., Calonge. I., \& Martínez-Arias, R. (2018). Posttraumatic growth in children and adolescents exposed to the 2010 earthquake in Chile and its relationship with rumination and posttraumatic stress symptoms. Journal of Happiness Studies, 19(5), 1505-1517. https://doi.org/10.1007/ s10902-017-9885-7

Andrades, M., García, F., Reyes, A., Martínez-Arias, R., \& Calonge. I. (2016). Psychometric properties of the posttraumatic growth inventory for children in Chilean population affected by the earthquake of 2010. American Journal of Orthopsychiatry, 86(6) 1505-1517. https://doi.org/10.1037/ort0000182

Barakat, L., Alderfer, M., \& Kazak, A. (2006). Posttraumatic growth in adolescent survivors of cancer and their mothers and fathers. Journal of Pediatric Psychology, 31, 413-419. https://doi. org/10.1093/jpepsy/jsj058

Bollen, K. \& Long, J. S. (1994). Testing structural equation models. Newbury Park, CA: Sage.

Calhoun, L. G., Cann, A., \& Tedeschi, R. G. (2010). The posttraumatic growth model: Sociocultural considerations. En: T. Weiss \& R. Berger (Eds.), Posttraumatic growth and culturally competent practice (pp.1-14). New York: Wiley \& Sons. https://doi. org/10.1002/9781118270028.ch1

Calhoun, L. G., \& Tedeschi, R. G. (1999). Facilitating posttraumatic growth: A clinician's guide. Mahwah, N.J.: Lawrence Erlbaum. https://doi.org/10.4324/9781410602268 
Calhoun. L. G., \& Tedeschi, R. G. (2006). Handbook of posttraumatic growth: Research and practice. London: Lawrence Erlbaum.

Cann, A., Calhoun, L. G., Tedeschi, R. G., Triplett, K., Vishnevsky, T., $\&$ Lindstrom, C. (2011). Assessing posttraumatic cognitive processes: The event related rumination inventory. Anxiety, Stress, \& Coping, 24(2), 137-156. https://doi.org/10.1080/10615806 .2010 .529901

Casullo, M. \& Fernández L., M. (2001). Estrategias de afrontamiento en estudiantes adolescentes. Revista del Instituto de Investigaciones de la Facultad de Psicología, 6(1), 25-49.

Cho, D. \& Park, C. L. (2013). Growth following trauma: Overview and current status. Terapia Psicológica, 31(1), 69- 79. https:// doi.org/10.4067/S0718-48082013000100007

Cohen, J.A., \& Gadassi, R. (2009). Posttraumatic stress disorder in young children exposed to terrorism: Validation of the alternative diagnostic criteria. Journal of the Child \& Adolescent Trauma, 2(4), 229-241. https://doi.org/10.1080/19361520903317295

Compas, B. E., Connor-Smith, J. K., Saltzman, H., Thomsen, A. H. \& Wadsworth, M. E. (2001). Coping with stress during childhood and adolescence: Problems progress and potential in theory and research. Psychological Bulletin, 127, 87-127. https://doi. org/10.1037//0033-2909.127.1.87

Cryder, C., Kilmer, R., Tedeschi, R. G., \& Calhoun, L. G. (2006). An exploratory study of posttraumatic growth in children following a natural disaster. American Journal of Orthopsychiatry, 76(1), 65-69. https://doi.org/10.1037/0002-9432.76.1.65

Dávila, B. \& Guarino, L. (2001). Fuentes de estrés y estrategias de afrontamiento en escolares y venezolanos. Interamerican Journal of Psychology, 35, 97-112

García, F., Duque, A. \& Cova, F. (2017). The four faces of rumination to stressful events: A psychometric analysis. Psychological Trauma: Theory, Research, Practice, and Police, 9(6), 758-765. https://doi. org/10.1037/tra0000289 
González, C. (2004). La psicología positiva: un cambio en nuestro enfoque patológico clásico. Liberabit, 10, 82-88.

González, R., Montoya, I., Casullo, M. M. \& Bernabéu, J. (2002). Relación entre estilos estrategias de afrontamiento y bienestar psicológico en adolescentes. Psicothema, 14, 363-368.

Hafstad, G., Gil Rivas, V., Kilmer, R., \& Raeder, S. (2010). Posttraumatic growth among Norwegian children and adolescents following a natural disaster. American Journal of Orthopsychiatry, 80, 248-257. https://doi.org/10.1111/j.1939-0025.2010.01028.x

Hampel, P. \& Petermann, F. (2006). Perceived stress, coping, and adjustment in adolescents. Journal of Adolescent Health, 38, 409-415. https://doi.org/10.1016/j.jadohealth.2005.02.014

Helgeson, V., Reynolds, K., \& Tomich, P. (2006). A metaanalytic review of benefit finding and growth. Journal of Consulting and Clinical Psychology, 74, 797-816. https://doi. org/10.1037/0022-006X.74.5.797

Janoff-Bulman, R. (2006). Schema-change perspectives on posttraumatic growth. In L. G. Calhoun \& R. G. Tedeschi (Eds.), Handbook of posttraumatic growth: Research and practice (pp. 81-99). Mahwah, NJ: Erlbaum.

Kilmer, R. P., Gil-Rivas, V., Tedeschi, R. G., Cann, A., Calhoun, L. G., Buchanan, T., \& Taku, K. (2009). Use of the revised posttraumatic growth inventory for children. Journal of Traumatic Stress, 22(3), 248-253. https://doi.org/10.1002/jts.20410

Kilmer, R. P., \& Gil-Rivas, V. (2010). Exploring posttraumatic growth in children impacted by hurricane Katrina: Correlates of the phenomenon and developmental considerations. Child Development, 81(4), 1211-1227. https://doi. org/10.1111/j.1467-8624.2010.01463.x

Kun. P., Han, S. C., Chen, X. C, \& Yao, L (2009). Prevalence and risk factors for posttraumatic stress disorder: A cross-sectional study among survivors of the Wenchuan 2008 earthquake in China. Depression and Anxiety, 26(12), 1134-40. https://doi. org/10.1002/da.20612 
La Greca, A. M., \& Prinstein, M. J. (2002). Hurricanes and tornadoes. En A. M. La Greca, W. K. Silverman, E. M. Vernberg, \& M. C. Roberts (Eds.), Helping children cope with disasters and terrorism (pp. 107-138). Washington, DC: American Psychological Association. https://doi.org/10.1037/10454-005

Lazarus, R. S. (1966). Psychological Stress and the Coping Process. New York, NY: McGraw-Hill.

Lazarus, R. S. \& Folkman, R. S. (1984). Stress appraisal and coping. New York, NY: Springer.

Leiva, M. (2011). Impacto psicosocial de terremotos y tsunamis. Reflexiones basadas en investigaciones posteriores al 27-F chileno. Revista Sociedad, Ciudad y Territorio, 2, 1-12.

Linley, P. A. \& Joseph, S. (2004). Positive change following trauma and adversity: A review. Journal of Traumatic Stress, 17, 11-21. https://doi.org/10.1023/B:JOTS.0000014671.27856.7e

Meyerson, D. A., Grant, K. E., Carter, J. S., \& Kilmer, R. P. (2011). Posttraumatic growth among children and adolescents: A systematic review. Clinical Psychology Review, 31(6), 949-964. https://doi.org/10.1016/j.cpr.2011.06.003

Morales, F., Trianes, M., Blanca, M., Miranda, J., Escobar, M., \& Fernández, F. (2012). Escala de afrontamiento para niños (EAN): Propiedades psicométricas. Anales de Psicología, 28(2), 475-483. https://doi.org/10.6018/analesps.28.2.136221

Norris, F., \& Elrod, C. (2006). Psychosocial consequences of disaster. A review of past research. En F. Norris, S. Galea, M. Friedman, \& P. Watson (Eds.), Methods for disaster mental health research (pp. 3-19). New York: Guilford Press.

Páez, D., Vázquez, C., Bosco, S., Gasparre, A., Iraurgi, I., \& Sezibera, V. (2011). Crecimiento post estrés y post trauma: Posibles aspectos positivos y beneficiosos de la respuesta a los hechos traumáticos. En D. Páez, C. Beristain, J. González, N. Basabe \& J. De Rivera (Eds.). Superando la violencia colectiva y construyendo cultura de paz (pp. 311-339). Madrid: Fundamentos. 
Park, C. L., \& Ai, A. L. (2006). Meaning making and growth: New directions for research on survivors of trauma. Journal of Loss and Trauma, 11, 389-407. https://doi. org/10.1080/15325020600685295

Rochanakorn, K. (2007). IASC guidelines on mental health and psychosocial support in emergency settings. Geneva, Switzerland: IASC

Salter, E., \& Stallard, P. (2004). Posttraumatic growth in child survivors of a road traffic accident. Journal of Traumatic Stress, 17(4), 335-340. https://doi.org/10.1023/B:JOTS.0000038482.53911.01

Schroevers M., Helgeson V., Sanderman, R., \& Ranchor A. (2010). Type of social support matters for prediction of posttraumatic growth among cancer survivors. Psychooncology. 19(1), 46-53. https://doi.org/10.1002/pon.1501

Siqveland, J., Nygaard, E., Hussain, A., Tedeschi, R. G., \& Heir, T. (2015). Posttraumatic growth, depression and posttraumatic stress in relation to quality of life in tsunami survivors: A longitudinal study. Health and Quality of Life Outcomes, 13(1), 13-18. https://doi.org/10.1186/s12955-014-0202-4

Taku, K., Cann, A., Tedeschi, R. G., \& Calhoun, L. G. (2009). Intrusive versus deliberate rumination in posttraumatic growth across US and Japanese samples. Anxiety, Stress \& Coping, 22(2), 129-136. https://doi.org/10.1080/10615800802317841

Tedeschi, R. G. \& Calhoun, L. G. (2004). Posttraumatic growth: Conceptual foundations and empirical evidence. Psychological Inquiry, 15(1), 1-18. https://doi.org/10.1207/ s15327965pli1501_01

Turner-Sack A., Menna R., Setchell S., Maan C., \& Cataudella D. (2012). Posttraumatic growth, coping strategies, and psychological distress in adolescent survivors of cancer. Oncology Nursing Forum, 43(1), 48-56. https://doi.org/10.1188/16. ONF.48-56

United States Geological Survey (2015). The mw 8.3 September 16, 2015 Coquimbo, Chile. Earthquake and associated aftershock sequence. 
Denver, CO: USGS. Recuperado de https://earthquake.usgs. gov/static/lfs/learn/Coquimbo_Educational_Slides.pdf Vernberg, E. M., Silverman, W. K., La Greca A. M., \& Prinstein, M (1996). Prediction of posttraumatic stress symptoms in children after hurricane Andrew. Journal of Abnormal Psychology, 105(2), 237-248. https://doi.org/10.1037/0021-843X.105.2.237

Vishnevsky, T., Cann, A., Calhoun, L.G., Tedeschi, R.G., \& Demakis, G.J. (2010). Gender differences in self-reported posttraumatic growth: A metaanalysis. Psychology of Women Quarterly, 34, 110-120. https://doi.org/10.1111/j.1471-6402.2009.01546.x

Wright, M., Banerjee, R., Hoek, W., Rieffe, C. \& Novin, S. (2010). Depression and social anxiety in children: Differential links with coping strategies. Journal of Abnormal Child Psychology, 38, 405-419. https://doi.org/10.1007/s10802-009-9375-4

Yu, X., Lau, J., Zhang, J., Mak, W., Choi, K., Lui, W., Zhang, J., \& Chan, E. (2010). Posttraumatic growth and reduced suicidal ideation among adolescents at month 1 after the sichuan earthquake. Journal of Affective Disorders, 123, 327-331. https:// doi.org/10.1016/j.jad.2009.09.019

Zoellner T. \& Maercker A. (2006). Posttraumatic growth in clinical psychology: A critical review and introduction of a two component model. Clinical Psychology Review, 26, 626-653. https://doi.org/10.1016/j.cpr.2006.01.008

Recibido: 16/1/2019

Revisado: 18/07/2020

Aceptado: 20/07/2020 